\title{
Velocidade sistólica máxima e indice de resistência de artérias fetais durante a segunda metade da gestação
}

\author{
Peak systolic velocity and resistance index of fetal arteries during the second half of pregnancy
}

Antonio Gadelha da Costa ${ }^{1}$, Francisco Mauad Filho ${ }^{2}$, Patricia Spara ${ }^{3}$, Procópio de Freitas ${ }^{4}$, Geraldo Duarte ${ }^{5}$, Patrícia El Beitune ${ }^{6}$, Eduardo Barreto Gadelha ${ }^{7}$

\section{RESUMO}

Objetivo: avaliar a velocidade sistólica máxima (VSM) e o índice de resistência (IR) nas artérias fetais cerebral média (ACM), aorta supra-renal (ASR), aorta infra-renal (AIR) e artéria umbilical (AU), entre a $22^{\text {a }}$ e a $38^{\text {a }}$ semana de gestação. Métodos: estudo prospectivo no qual foram avaliados os parâmetros de 33 fetos normais na $22^{\mathrm{a}}, 26^{\mathrm{a}}, 30^{\mathrm{a}}, 34^{\mathrm{a}}$ e $38^{\mathrm{a}}$ semana de gestação. Foram incluídas gestações únicas, sem doenças e complicações e as que concordaram em participar do estudo. Os critérios de exclusão foram malformações fetais, descontinuidade do seguimento aos exames e mães usuárias de fumo, álcool e drogas ilícitas. Os exames ultra-sonográficos foram realizados por único observador. Para a aquisição do traçado dopplervelocimétrico na ACM, ASR, AIR e AU, o volume de amostra foi de 1 a $2 \mathrm{~mm}$, colocado no centro das artérias. O ângulo de insonação foi de $5^{\circ}$ a $19^{\circ}$, na ACM, inferior a $45^{\circ}$ na ASR e AIR e inferior a $60^{\circ}$ na AU. Utilizamos filtro de parede de 50-100 Hz. O cálculo dos parâmetros foi realizado automaticamente, com a imagem congelada, tendo sido acionadas três medidas. O resultado final foi obtido pela média aritmética dos três valores. A análise estatística foi realizada pela análise de variância (ANOVA), teste post hoc de Bonferroni, coeficiente de correlação de Pearson e análise de regressão. O nível de significância foi $\mathrm{p}<0,05$ em todas as análises. Resultados: a VSM aumentou de 26,3 para 57,7 cm/s na ACM, entre a 22 a e a $38^{a}$ semana de gestação ( $\left.<<0,05\right)$. Na ASR e na AIR, a VSM aumentou, entre a 22ª e a 34ª semana de gestação, de 74,6 e 59,0 $\mathrm{cm} / \mathrm{s}$ para 106,0 e $86,6 \mathrm{~cm} / \mathrm{s}$, respectivamente $(\mathrm{p}<0,05)$. Na AU, a VSM aumentou entre a $22^{\mathrm{a}}$ e a $34^{\mathrm{a}}$ semana de gestação, porém diminuiu de 55,5 para $46,2 \mathrm{~cm} / \mathrm{s}$ entre a $34^{\mathrm{a}}$ e a $38^{\mathrm{a}}$ semana de gestação. O IR, na ACM, foi menor na $22^{\mathrm{a}}(0,81)$ e $38^{\mathrm{a}}$ semana de gestação $(0,75)$ e maior $(0,85)$ na $26^{\mathrm{a}}$ semana $(\mathrm{p}<0,05)$. Na ASR, os valores do IR foram estáveis em todas as semanas e na maioria delas na AIR ( $\mathrm{p}>0,05)$. Na AU, o IR diminuiu de 0,69 para 0,56 , entre a $22^{\mathrm{a}}$ e a $38^{\mathrm{a}}$ semana gestacional $(\mathrm{p}<0,05)$. Conclusão: em fetos normais, na segunda metade da gestação, a VSM aumenta na ACM, ASR e AIR, diminuindo na AU entre a $34^{\mathrm{a}}$ e a $38^{\mathrm{a}}$ semana de gestação. O IR é menor na $22^{\mathrm{a}}$ e $38^{\mathrm{a}}$ semana de gestação na ACM, diminui entre a $22^{\mathrm{a}}$ e a $38^{\text {a }}$ semana na AU e é constante na maioria das semanas gestacionais na ASR e AIR.

PALAVRAS-CHAVE: Velocidade do fluxo sangüíneo; Resistência vascular; Feto/irrigação sangüínea; Fluxometria por laser-doppler

\section{ABSTRACT}

Purpose: to assess peak systolic velocity (PSV) and the resistance index (RI) in the middle cerebral artery (MCA), suprarenal aorta (SRA) and infrarenal aorta (IRA) of the fetus and in the umbilical artery (UA) between the 22nd and 38th week of gestation. Methods: a prospective study which evaluated the parameters of 33 normal fetuses in the $22^{\text {nd }}, 26$ th, $30^{\text {th }}$, and 38 th week of gestation. Pregnant women with a singleton fetus with no diseases or complications and who agreed to participate were included in the study. Exclusion criteria were fetal malformations, discontinuation of prenatal care visits and mothers who smoked, used alcohol or illicit drugs. Ultrasound examinations were performed by a single observer. For the acquisition of the Doppler velocimetry tracing in the MCA, SRA, IRA and UA, the sample volume was 1 to $2 \mathrm{~mm}$, placed in the center of the arteries. The insonation angle was $5^{\circ}$ to $19^{\circ}$ in the MCA, below $45^{\circ}$ in the SRA and IRA, and less than $60^{\circ}$ in the UA. We used a wall filter of 50-100 Hz. The parameters were calculated automatically with the frozen image, three measurements being made. The final result was obtained by the arithmetic mean of the three values. Data were analyzed by analysis of

Trabalho realizado no Hospital das Clínicas da Faculdade de Medicina de Ribeirão Preto da Universidade de São Paulo - USP - Ribeirão Preto (SP) - Brasil. 1 Professor Adjunto do Departamento Materno Infantil da Universidade Federal de Campina Grande - UFCG - Campina Grande (PB) - Brasil.

2 Professor Associado do Departamento de Ginecologia e Obstetrícia da Faculdade de Medicina de Ribeirão Preto, Universidade de São Paulo - USP Ribeirão Preto (SP) - Brasil.

3 Professor Adjunto do Departamento Materno Infantil da Universidade Federal de Campina Grande - UFCG - Campina Grande (PB) - Brasil.

4 Professor da Escola de Ultra-Sonografia Ribeirão Preto (SP) - Brasil.

5 Professor Titular do Departamento de Ginecologia e Obstetrícia da Faculdade de Medicina de Ribeirão Preto, Universidade de São Paulo - USP Ribeirão Preto (SP) - Brasil.

6 Doutor pelo Departamento de Ginecologia e Obstetrícia da Faculdade de Medicina de Ribeirão Preto, Universidade de São Paulo - USP - Ribeirão Preto (SP) - Brasil.

7 Residente do Hospital Barão de Lucena - Recife (PE) - Brasil.

Correspondência: Antonio Gadelha da Costa

Rua José da Silva, 726 - Apto. 34 - Jardim Paulista - 14090-040 - Ribeirão Preto - SP - e-mail: iggadelha@ig.com.br

Recebido em: 2/9/2005 Aceito com modificações em: 24/6/2005

Rev Bras Ginecol Obstet. 2005;27(7):387-92 
variance (ANOVA), post hoc Bonferroni test, Pearson's correlation, and regression analysis. The level of significance was set at $\mathrm{p}<0.05$ in all analyses. Results: PSV increased from 26.3 to $57.7 \mathrm{~cm} / \mathrm{s}$ in the MCA between the $22 \mathrm{nd}$ and the 38 th week of gestation $(\mathrm{p}<0.05)$. In the SRA and in the IRA, PSV increased between the 22nd and 34th week of gestation, from 74.6 and $59.0 \mathrm{~cm} / \mathrm{s}$ to 106.0 and $86.6 \mathrm{~cm} / \mathrm{s}$, respectively $(\mathrm{p}<0.05)$. In the UA, PSV increased between the $22 \mathrm{nd}$ and the 34 th week of gestation, but decreased from 55.5 to $46.2 \mathrm{~cm} / \mathrm{s}$ between the 34 th and the 38 th week of gestation. In the MCA, the RI was lower in the 22nd (0.81) and 38th week of gestation (0.75) and higher (0.85) in the 26th week $(\mathrm{p}<0.05)$. In the SRA, the RI values were stable in all weeks and in the IRA they were stable in most weeks ( $>0.05)$. In the UA, RI decreased from 0.69 to 0.56 between the $22 \mathrm{nd}$ and 38 th week of gestation $(\mathrm{p}<0.05)$. Conclusion: in normal fetuses, in the second half of gestation PSV increased in the MCA, SRA and IRA, decreasing in the UA between the 34th and 38th week of gestation. RI was lower in the $22 \mathrm{nd}$ and 38 th weeks of gestation in the MCA, decreased between the 22nd and the 38th week in the UA, and was constant in most of the gestational weeks in the SRA and IRA.

Keywords: Blood flow velocity; Vascular resistance; Fetus/blood suply; Laser-doppler flowmetry

\section{Introdução}

Com o início da função cardiaca embrionária, entre a $5^{\text {a }}$ e a $6^{a}$ semana de gestação, pode-se observar velocidade de fluxo sanguíneo no coração e no eixo longitudinal do embrião, em topografia da aorta. O aumento da velocidade de fluxo sanguíneo e dos batimentos cardiacos embrionários está relacionado com a maturidade do embrião. Com 5 a 6 semanas de gestação, os batimentos embrionários são 100 a $130 \mathrm{bpm}$, aumentando para $137( \pm 19) \mathrm{bpm}$, com 7 a 8 semanas ${ }^{1}$.

O sistema vascular fetal é composto de compartimentos vasculares. A aorta descendente é subdividida em aorta torácica ou supra-renal (ASR) e aorta abdominal ou infra-renal (AIR). A aorta fetal ascendente supre de sangue o cérebro e o tecido miocárdico. A aorta fetal descendente supre órgãos do abdome e as artérias ilíacas externa e interna. As artérias iliacas externas irrigam a parte inferior do corpo ${ }^{2}$.

A ultra-sonografia baseada no efeito Doppler possibilitou a avaliação hemodinâmica não invasiva do feto. É utilizada na avaliação da resposta fetal à hipóxia, por meio da dopplervelocimetria na artéria cerebral média (ACM) do feto e no ducto venoso, como também nos fetos com restrição de crescimento intra-uterino, pelo estudo dopplervelocimétrico na artéria umbilical (AU) ${ }^{3}$. Na atualidade, o diagnóstico de anemia fetal grave é realizado pela dopplervelocimetria na ACM do feto, evitando-se a realização de procedimentos invasivos ${ }^{4,5}$.

Para a observação de complicações na circulação fetal e na adaptação hemodinâmica em fetos de risco é obrigatório reconhecer mudanças hemodinâmicas fetais durante a gestação normal ${ }^{6}$. Trabalhos recentes descrevem a hemodinâmica fetal por meio da dopplervelocimetria, demonstrando a interação entre o débito cardíaco e o sistema arterial e venoso fetal ${ }^{6}$, e por modelo artificial, que simula a circulação do feto ${ }^{2}$.

Seguindo essa linha de investigação, o objetivo deste trabalho foi avaliar os valores da velocidade sistólica máxima (VSM) e índice de resistência (IR) nas artérias fetais cerebral média, umbilical, aorta torácica e abdominal entre a $22^{\text {a }}$ e a $38^{\text {a }}$ semana de gestação.

\section{Métodos}

Realizamos estudo prospectivo longitudinal, com análise dos parâmetros hemodinâmicos, em 33 fetos de gestantes normais, com idade entre 15 e 41 anos. O trabalho foi aprovado pelo Comitê de Ética em Pesquisa do Hospital das Clínicas da Faculdade de Medicina de Ribeirão Preto da Universidade de São Paulo.

O cálculo amostral foi realizado por meio do programa GraphPad StatMate 1.01i, baseado na diferença de média entre o maior e o menor valor de cada variável dependente, utilizando o maior desvio padrão. Para poder de teste de $80 \%$ e nivel de significância $(p=0,05)$ foi indicado avaliar 25 pacientes para a VSM e 30 pacientes para o IR.

Todas as pacientes tinham gestação única, idade gestacional entre a $22^{\mathrm{a}}$ e a $38^{\mathrm{a}}$ semana, calculada pela data da última menstruação e/ou avaliação ultra-sonográfica do comprimento cabeçanádega entre a $8^{\mathrm{a}}$ e a $12^{\mathrm{a}}$ semana de gestação. Não eram portadoras de enfermidades maternas associadas e/ou próprias da gestação, como também não eram usuárias de fumo, álcool ou drogas. Os fetos não eram portadores de malformação fetal e apresentaram crescimento adequado.

O primeiro exame ultra-sonográfico foi realizado entre a $8^{\mathrm{a}}$ e a $12^{\mathrm{a}}$ semana de gestação, no intuito de se determinar a idade gestacional pelo comprimento cabeça-nádega. Os exames subseqüentes foram feitos a partir da $22^{\mathrm{a}}$ semana, com intervalo de quatro semanas, até a $38^{\mathrm{a}}$ semana 
de gestação. Todos os fetos foram avaliados na $22^{\mathrm{a}}$, $26^{\mathrm{a}}, 30^{\mathrm{a}}, 34^{\mathrm{a}}$ e $38^{\mathrm{a}}$ semana de gestação.

Os exames foram realizados por único observador (AGC), utilizando aparelho ultrasonográfico modelo Image Point 1800 (Hewlett Packard), transdutor multifreqüencial $(5,0,7,5$ e $10 \mathrm{MHz}$ ). A paciente foi posicionada em decúbito dorsal, posição semi-sentada, em ângulo de $15^{\circ} \mathrm{e}$ $30^{\circ}$ entre a mesma e a mesa de exame, evitandose, deste modo, a compressão da veia cava ${ }^{7}$. A seguir, procedia-se à formação das imagens e ao mapeamento pelo efeito Doppler. As aferições dopplervelocimétricas foram realizadas com os fetos em repouso, na ausência de movimentos respiratórios e com freqüência cardiaca fetal normal (120$160 \mathrm{bpm})$.

Para a aquisição do traçado dopplervelocimétrico da ACM realizamos, inicialmente, corte axial da cabeça fetal no nível do tálamo e do cavo do septo pelúcido. Em seguida moviamos o transdutor até observarmos, por inclinação cranial, o polígono de Willis e a pulsação das duas artérias cerebrais médias. Por meio do Doppler de amplitude visualizamos a maior parte do trajeto da ACM. Ao acionar, simultaneamente, o Doppler espectral obtivemos as ondas de velocidade de fluxo. O volume de amostra foi calibrado para 1 a $2 \mathrm{~mm}$ e posicionado no centro da ACM, próximo da calota craniana, antes da sua bifurcação ${ }^{8}$. Para se evitarem artefatos nos fluxogramas, fizemos a otimização do ângulo entre o feixe dopplervelocimétrico e a trajetória do fluxo para valores entre $5^{0}$ e $19^{\circ}$, nos quais os erros para as velocidades sistólicas e diastólicas são despreziveis ${ }^{9}$. Em todos os vasos, o filtro de parede foi ajustado na freqüência de $50-100 \mathrm{~Hz}$, para evitar a possibilidade de perda do componente diastólico ${ }^{10}$.

O traçado dopplervelocimétrico na aorta fetal supra-renal e infra-renal foi obtido por meio de corte sagital abrangendo o tórax e abdome do feto, no qual, por meio do Doppler de amplitude, visualizamos a aorta fetal em toda sua extensão. O volume de amostra foi de 1 a $2 \mathrm{~mm}$, posicionado no centro da aorta avaliada. O ângulo entre o feixe sonoro e a trajetória do fluxo foi abaixo de $45^{\circ}$, no qual os erros para as velocidades sistólicas e diastólicas são inferiores a 9\% ${ }^{9}$.

Os registros das velocidades de fluxo na AU foram obtidos por meio de corte sagital do cordão umbilical, 3 a $5 \mathrm{~cm}$ da inserção placentária, no qual, pelo Doppler colorido, visualizamos as duas artérias e a veia umbilical. Acionando-se simultaneamente o Doppler espectral, obtivemos os sonogramas dopplervelocimétricos. Devido a dificuldade técnica, o ângulo entre o feixe dopplervelocimétrico e a trajetória do fluxo, na AU, foi abai- xo de $60^{\circ}$, valor aceitável para vasos com dificuldade técnica ${ }^{11}$. O volume de amostra foi de 1 a 2 $\mathrm{mm}$, posicionado no centro da artéria.

Os cálculos dos parâmetros foram realizados automaticamente, com a imagem congelada, tendo sido obtidas três medidas. O resultado final foi obtido pela média aritmética dos três valores.

Os recém-nascidos foram avaliados por neonatologista que determinou índice de Capurro et al. ${ }^{12}$ somático, sendo considerados adequados para a idade gestacional. Além disso, não havia alterações nos diversos sistemas, inclusive o cardiovascular. O índice de Apgar foi superior a sete, no primeiro e quinto minutos. O peso variou de 2.800 a $3.930 \mathrm{~g}$.

As variáveis dependentes foram a VSM e o IR nas artérias, ACM, AU, ASR e AIR. A variável independente foi a idade gestacional entre a $22^{\mathrm{a}} \mathrm{e}$ a $38^{a}$ semana de gestação. A análise estatística foi realizada, por meio da análise de variância (ANOVA), teste post hoc de Bonferroni, coeficiente de correlação de Pearson e análise de regressão. Em todas as análises estatísticas foi estabelecido como nivel de significância $\mathrm{p}<0,05$.

Utilizamos a ANOVA para verificarmos se as variáveis independentes (idade gestacional em semanas) produziriam modificações nas variáveis dependentes (parâmetros dopplervelocimétricos). O teste post hoc de Bonferroni foi realizado para comparar duas variáveis dependentes, tornando mais claras as diferenças encontradas pelo método ANOVA.

As correlações foram realizadas entre as variáveis independentes e dependentes para analisarmos o indicador de força da relação linear existente entre essas duas variáveis. A análise de regressão foi utilizada para que pudéssemos desenvolver uma equação para prever valores das variáveis dependentes em função das variáveis independentes

\section{Resultados}

A VSM aumentou progressivamente, entre a $22^{\text {a }}$ e a $38^{\mathrm{a}}$ semana de gestação, na ACM. O valor do percentil 50 na $22^{\mathrm{a}}$ semana foi 26,3 e na $38^{\mathrm{a}}$ semana $57,7 \mathrm{~cm} / \mathrm{s}(\mathrm{p}<0,001$ - ANOVA, $\mathrm{p}<0,05-$ teste post hoc de Bonferroni) (Tabela 1). Na ASR e AIR a VSM aumentou progressivamente até a $34^{\mathrm{a}}$ semana, e os valores do percentil 50 foram 106 e $86,6 \mathrm{~cm} / \mathrm{s}$, respectivamente, mantendo-se constante entre a $34^{\mathrm{a}}$ e a $38^{\mathrm{a}}$ semana de gestação $\left(\mathrm{p}<0,001\right.$ - ANOVA; $\mathrm{p}<0,05$ até a $34^{\mathrm{a}}$ semana gestacional e $\mathrm{p}>0,05$ entre a $34^{\mathrm{a}}$ e a $38^{\mathrm{a}}$ semana de 
gestação - teste post hoc de Bonferroni) (Tabela 1). Na AU observamos aumento da VSM entre a $22^{\text {a }} \mathrm{e}$ a $34^{a}$ semana de gestação. Entretanto, houve diminuição de $55,5 \mathrm{~cm} / \mathrm{s}$, no percentil 50 , para 46,2 $\mathrm{cm} / \mathrm{s}$ entre a $34^{\mathrm{a}}$ e a $38^{\mathrm{a}}$ semana $(\mathrm{p}<0,001$ - Anova, $\mathrm{p}<0,05$ teste post hoc de Bonferroni) (Tabela 1). Observamos forte correlação linear entre a idade gestacional e a VSM, na ACM $(r=0,77, p<0,001$ correlação de Pearson), ao passo que, na ASR, AIR e AU, obtivemos correlação imperfeita positiva $(\mathrm{r}=0,65, \mathrm{r}=0,58$ e $\mathrm{r}=0,58$, respectivamente - correlação de Pearson). Os números apresentados significam o resultado da aplicação da equação da reta. Para a VSM na ACM, ASR, AIR e AU, as equações da reta foram, respectivamente, $\mathrm{ACM}=-$ $21,47+2,16 \times$ semana; ASR $=-55,48+7,84 \times$ semana $-0.09 \times$ semana $^{2}$; AIR $=-117,73+11,65 \times$ semana $-0,17 \times$ semana $^{2}$ e $A U=-34,07+4,90 \times$ semana $-0,07 \times$ semana $^{2}$.

Tabela 1 - Percentis 5, 50 e 95 da velocidade sistólica máxima (VSM) nas artérias fetais cerebral média (ACM), aorta supra-renal (ASR), aorta infra-renal (AIR) e artéria umbilical (AU), de acordo com a idade gestacional (IG) em semanas.

\begin{tabular}{|c|c|c|c|c|c|c|c|c|c|c|c|c|}
\hline \multicolumn{4}{|c|}{$\mathrm{ACM}$} & \multicolumn{3}{|c|}{ ASR } & \multicolumn{3}{|c|}{ AIR } & \multicolumn{3}{|c|}{$A U$} \\
\hline IG & P5 & P50 & P95 & P5 & P50 & P95 & P5 & P50 & P95 & P5 & P50 & P95 \\
\hline
\end{tabular}

22 16,7 26,3 40,8 52,5 74,6 97,4 42,2 $59,0 \quad 77,6 \quad 26,6 \quad 35,4 \quad 67,0$

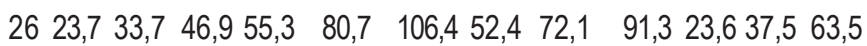
$3032,143,8 \quad 55,172,1 \quad 100,0 \quad 119,956,7 \quad 79,0 \quad 116,2 \quad 29,847,6 \quad 99,1$ $3429,052,973,072,1 \quad 106,0^{*} \quad 147,560,4 \quad 86,6^{*} \quad 133,7 \quad 36,155,5 \quad 81,6$ $3833,157,7^{*} 97,884,2 \quad 107,0^{* *} 136,960,0 \quad 85,8^{* *} 110,526,746,2^{*} 71,1$ 33 fetos em cada idade gestacional. $p<0,001$ para a análise de todas as semanas (ANOVA). ${ }^{*} p<0,05,{ }^{* *} p>0,05$ - teste post hoc de Bonferroni.

$\mathrm{Na}$ ACM , o IR foi menor na $22^{\mathrm{a}}$ e na $38^{\mathrm{a}}$ semana de gestação e maior na $26^{\mathrm{a}}$ semana de gestação. Os valores do percentil 50 foram, respectivamente, $0,81,0,75$ e $0,85(\mathrm{p}<0,001$ - ANOVA, $\mathrm{p}<0,05$ teste Post hoc de Bonferroni) (Tabela 2). A correlação entre idade gestacional e o IR foi imperfeita positiva $(r=0,27-p<0,001$ - correlação de Pearson). Na ASR, o IR não variou no periodo estudado, não havendo, portanto, correlação $(\mathrm{p}=0,73$ ANOVA) (Tabela 2). No entanto, na AIR observamos diferença entre as semanas, com diminuição de 0,75 para 0,71 entre a $22^{\mathrm{a}}$ e a $26^{\mathrm{a}}$ semana de gestação (valores do percentil $50, p<0,001-$ ANOVA, $\mathrm{p}<0,05$ teste post hoc de Bonferroni, $\mathrm{r}=0,25$ - correlação de Pearson) (Tabela 2). Houve diminuição progressiva do IR, na $\mathrm{AU}$, entre a $22^{\mathrm{a}}$ semana e a $38^{\mathrm{a}}$ semana de gestação, cujos valores diminuíram de 0,69 , no percentil 50 , para 0,56 ( $\mathrm{p}<0,001$ - Anova, $\mathrm{p}<0,05$ - teste post hoc de Bonferroni, Tabela 2), com correlação imperfeita positiva ( $r=0,6-p<0,001$ - correlação de Pearson). Os números apresentados significam o resultado da aplicação da equação da reta. Para o IR na ACM, AIR e AU, as equações da reta foram, respectivamente, $A C M=0,02+0,06 \times$ semana $-0,001 \times$ semana $^{2}$; AIR $=1,08-0,021 \times$ semana $+0,0003 \times$ semana $^{2}$ e $A U=0,87-0,009 \times$ semana.

Tabela 2 - Percentis 5, 50 e 95 do índice de resistência nas artérias fetais cerebral média (ACM), aorta supra-renal (ASR), aorta infra-renal (AIR) e artéria umbilical (AU), de acordo com a idade gestacional (IG) em semanas.

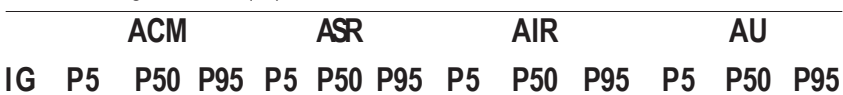

\begin{tabular}{lllllllllllll}
\hline 2 & 0,72 & 0,81 & 0,97 & 0,77 & 0,83 & 0,89 & 0,67 & 0,75 & 0,85 & 0,59 & 0,69 & 0,78
\end{tabular}

$\begin{array}{lllllllllllllllllllll} & 0,76 & 0,85 & 0,97 & 0,73 & 0,82 & 0,87 & 0,61 & 0,71^{*} & 0,81 & 0,53 & 0,64^{*} & 0,74\end{array}$

$\begin{array}{llllllllllllllll} & 0,73 & 0,83^{*} & 0,95 & 0,72 & 0,81 & 0,93 & 0,59 & 0,72 & 0,81 & 0,50 & 0,62^{* *} & 0,74\end{array}$

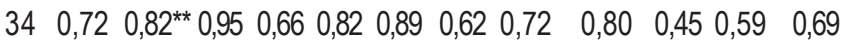
$\begin{array}{lllllllllllllll} & 38 & 0,67 & 0,75^{*} & 0,84 & 0,7 & 0,82 & 0,87 & 0,64 & 0,71 * * & 0,80 & 0,44 & 0,56 * & 0,64\end{array}$ 33 fetos em cada idade gestacional. $p<0,001$ para a análise de todas as semanas na ACM, AIR (ANOVA). $p>0,05$ para a análise de todas as semanas na ASR (ANOVA). ${ }^{*} p<$ $0,05,{ }^{* *} p>0,05$ - teste post hoc de Bonferroni.

\section{Discussão}

A velocidade do fluxo sanguíneo depende da resistência periférica, complacência vascular e inércia da corrente sanguínea e as modificações são relacionadas ao crescimento fetal ${ }^{13}$. Observamos que a VSM aumentou progressivamente entre a $22^{\mathrm{a}}$ e a $38^{\mathrm{a}}$ semana de gestação na ASR e AIR. Isso demonstra que na segunda metade da gestação ocorreu distribuição crescente de fluxo sanguíneo pela aorta fetal para o cérebro, fibra miocárdica, órgãos do abdome superior e inferior, artérias iliacas, membros superiores e inferiores, como também, leito placentário. Em fetos saudáveis a velocidade de fluxo sanguíneo na aorta descendente é alta, sendo que 50 a $60 \%$ do volume sanguíneo supre a placenta ${ }^{14}$.

Em situações de insuficiência placentária ocorre aumento da resistência placentária, comprometendo as trocas materno-fetais e o desenvolvimento do feto ${ }^{14}$. Verificamos que, entre a $22^{\text {a }}$ e a $38^{\text {a }}$ semana de gestação, a VSM manteve-se alta na aorta fetal e o IR, na AU, diminuiu progressivamente. Dessa forma, fetos saudáveis mantêm a velocidade de fluxo sanguíneo adequado na aorta para suprir órgãos vitais durante seu desenvolvimento, e resistência baixa no leito placentário para permitir trocas materno-fetais de oxigênio e nutrientes.

Um quarto do débito cardíaco fetal é distribuído para a placenta e $25 \%$ para os membros inferiores por meio das artérias ilíaca interna e ilíaca externa fetais, ramos da aorta fetal ${ }^{2}$. Observamos que entre a $34^{\mathrm{a}}$ e a $38^{\mathrm{a}}$ semana de gestação a VSM manteve-se constante na AIR e dimi- 
nuiu na AU. Inferimos, portanto, que nessa idade gestacional houve vasodilatação no sistema vascular placentário, tendo em vista a diminuição da VSM na AU. As trocas materno-fetais pela placenta foram mantidas, tendo em vista que, na $\mathrm{AU}$, o IR diminuiu progressivamente entre a $22^{\mathrm{a}} \mathrm{e}$ a $38^{a}$ semana de gestação. Nas gestações normais, após a $22^{\mathrm{a}}$ e a $24^{\mathrm{a}}$ semana de gestação, a velocidade de fluxo sanguíneo útero-placentário é constante e a resistência na artéria umbilical diminui, permanecendo constante na aorta descendente fetal ${ }^{15}$. No nosso estudo também observamos diminuição progressiva do IR na AU durante a gestação e manutenção desse índice na ASR entre a $22^{\mathrm{a}}$ e a $38^{\mathrm{a}}$ semana de gestação, como também, na maioria das semanas, na AIR. A resistência na aorta fetal descendente não acompanha a diminuição observada nas artérias umbilicais com o progredir da gestação, permanecendo constante até o termo. Isto ocorre devido ao aumento da resistência vascular dos vasos periféricos, comprovado pelo aumento do índice de pulsatilidade na artéria femoral fetal no final da gestação ${ }^{16}$.

O aumento progressivo da VSM na ASR do feto, encontrado em nosso estudo, e a diminuição progressiva do IR, na artéria renal fetal, ao longo da gestação ${ }^{17,18}$, são, entre outros fatores, fenômenos hemodinâmicos relacionados ao desenvolvimento do rim.

O suprimento de oxigênio e nutrientes para o desenvolvimento do cérebro fetal provém do fluxo sanguíneo da aorta ascendente, por meio do tronco braquiocefálico e carótida comum esquerda. O fluxo sanguíneo é impulsionado para esses troncos vasculares, após contração do ventrículo esquerdo, e posteriormente para a artéria carótida interna e artéria cerebral média, que irriga a maior parte de cada hemi-hemisfério cerebral ${ }^{13}$. Observamos que o suprimento sanguíneo para o cérebro fetal, entre a $22^{\mathrm{a}}$ e a $38^{\mathrm{a}}$ semana de gestação, foi crescente, tendo em vista que a VSM na ACM aumentou progressivamente ao longo da gestação. Esse dado também foi relatado por Kurmanavicius et al. ${ }^{19} \mathrm{e}$ Bahlmann et al. ${ }^{20}$. Verificamos que, enquanto a VSM aumentou progressivamente, na ACM, entre a $22^{\mathrm{a}}$ e a $38^{\mathrm{a}}$ semana de gestação, o IR aumentou e diminuiu, em função da idade gestacional. No terceiro trimestre da gestação, a impedância nas artérias fetais cerebral média e umbilical é menor, mantendo-se constante na aorta fetal descendente $^{14}$. No nosso estudo, o IR, na ACM, diminuiu entre a $34^{\mathrm{a}}$ e a $38^{\mathrm{a}}$ semana gestacional, sendo que seu valor foi menor na $38^{a}$ semana. Portanto, o suprimento de fluxo sanguíneo para o cérebro foi maior a partir da $34^{a}$ semana, período no qual ocorre maior maturidade cerebral ${ }^{21}$.
Concluímos que, em fetos normais, a velocidade sistólica máxima aumentou progressivamente na aorta supra-renal e infra-renal, entre a $22^{a}$ e a $38^{a}$ semana de gestação, diminuindo na artéria umbilical entre a $34^{\mathrm{a}}$ e a $38^{\mathrm{a}}$ semana gestacional. $\mathrm{O}$ indice de resistência foi menor na $22^{\mathrm{a}}$ e na $38^{\mathrm{a}}$ semana de gestação na artéria cerebral média, diminui entre a $22^{\mathrm{a}}$ e a $38^{\mathrm{a}}$ semana na artéria umbilical e permaneceu constante na maioria das semanas gestacionais na aorta supra-renal e infra-renal, considerando-se a $22^{\mathrm{a}}$ e a $38^{\mathrm{a}}$ semana gestacional.

\section{Referências}

1. Ji RP, Phoon CK, Aristizabal O, McGrath KE, Palis $\mathrm{J}$, Turnbull DH. Onset of cardiac function during early mouse embryogenesis coincides with entry of primitive erythroblasts into the embryo proper. Circ Res. 2003;92(2):133-5.

2. Myers LJ, Capper WL. A transmission line model of the human foetal circulatory system. Med Eng Phys. 2002;24(4):285-94.

3. Baschat AA, Gembruch U, Reiss I, Gortner L, Weiner CP, Harman CR. Relationship between arterial and venous Doppler and perinatal outcome in fetal growth restriction. Ultrasound Obstet Gynecol. 2000;16(5):407-13.

4. Mari G, Detti L, Oz U, Zimmerman R, Duerig P, Stefos T. Accurate prediction of fetal hemoglobin by Doppler ultrasonography. Obstet Gynecol. 2002;99(4):589-93.

5. Scheier M, Hernandez-Andrade E, Carmo A, Dezerega V, Nicolaides KH. Prediction of fetal anemia in rhesus disease by measurement of fetal middle cerebral artery peak systolic velocity. Ultrasound Obstet Gynecol. 2004;23(5):432-6.

6. Chang $\mathrm{CH}$, Chang $\mathrm{FM}$, Yu $\mathrm{CH}$, Liang RI, Ko HC, Chen HY. Systemic assessment of fetal hemodynamics by Doppler ultrasound. Ultrasound Med Biol. 2000;26(5):777-85.

7. Özeren M, Dinç H, Ekmen Ü, Senekayli C, Aydemir V. Umbilical and middle cerebral artery Doppler indices in patients with preeclampsia. Eur J Obstet Gynecol Reprod Biol. 1999;82(1):11-6.

8. Luzi G, Coata G, Caserta G, Cosmi EV, Di Renzo GC. Doppler velocimetry of different sections of the fetal middle cerebral artery in relation to perinatal outcome. J Perinat Med. 1996;24(4):327-34.

9. Burns PN. Hemodynamics. In: Taylor KJW, Burns PN, Wells PNT, editors. Clinical applications of Doppler ultrasound. 2nd ed. New York: Raven Press; 1995. p. 35-98. 
10. Mari G, Deter RL. Middle cerebral artery flow velocity waveforms in normal and small- for-gestational-age fetuses. Am J Obstet Gynecol. 1992;166(4):126270 .

11. Taylor KJ, Holland S. Doppler US. Part I. Basic principles, instrumentation, and pitfalls. Radiology. 1990;174(2):297-307.

12. Capurro H, Konichezky S, Fonseca D, CaldeyroBarcia R. A simplified method for diagnosis of gestational age in the newborn infant. J Pediatr. 1978;93(1):120-2.

13. Saburi Y, Mori A, Yasui I, Makino T, Iwabuchi M. Fetal aortic blood flow assessment from the relationship between fetal aortic diameter pulse and flow velocity waveforms during fetal development. Early Hum Dev. 2001;65(1):57-70.

14. Brezinka C. Fetal hemodynamics. J Perinat Med. 2001;29(5):371-80.

15. Ertan AK, Hendrik HJ, Tanriverdi HA, Bechtold M, Schmidt W. Fetomaternal Doppler sonography nomograms. Clin Exp Obstet Gynecol. 2003;30(4):211-6.

16. Arduini D, Rizzo G. Normal values of pulsatility index from fetal vessels: a cross-sectional study on 1556 healthy fetuses. J Perinat Med. 1990;18(3):165-72.
17.Akiyama M, Kuno A, Tanaka Y, Tanaka H, Hayashi $\mathrm{K}$, Yanagihara $\mathrm{T}$, et al. Comparison of alterations in fetal regional arterial vascular resistance in appropriate-for-gestational-age singleton, twin and triplet pregnancies. Hum Reprod. 1999;14(10):263543.

18. Andriani G, Persico A, Tursini S, Ballone E, Cirotti D, Lelli Chiesa P. The renal-resistive index from the last 3 months of pregnancy to 6 months old. BJU Int. 2001;87(6):562-4.

19. Kurmanavicius J, Streicher A, Wright EM, Wisser $\mathrm{J}$, Muller R, Royston P, et al. Reference values of fetal peak systolic blood flow velocity in the middle cerebral artery at 19-40 weeks of gestation. Ultrasound Obstet Gynecol. 2001;17(1):50-3.

20. Bahlmann F, Reinhard I, Krummenauer F, Neubert S, Macchiella D, Wellek S. Blood flow velocity waveforms of the fetal middle cerebral artery in a normal population: reference values from 18 weeks to 42 weeks of gestation. J Perinat Med. 2002;30(6):490-501.

21.Arnold SE, Trojanowski JQ. Human fetal hippocampal development: I. Cytoarchitecture, myeloarchitecture, and neuronal morphologic features. J Comp Neurol. 1996;367(2):274-92. 